\title{
The ways of using machine learning in dentistry
}

\author{
Monika Elżbieta Machoy ${ }^{1, A-F}$, Liliana Szyszka-Sommerfeld ${ }^{1, B, F}$, Andras Vegh ${ }^{2, E, F}$, Tomasz Gedrange ${ }^{3,4, E, F}$, Krzysztof Woźniak, ${ }^{1, E, F}$ \\ ${ }^{1}$ Department of Orthodontics, Pomeranian Medical University, Szczecin, Poland \\ ${ }^{2}$ Department of Orofacial Orthopaedics and Orthodontics, Heim Pal Children's Hospital, Budapest, Hungary \\ ${ }^{3}$ Division of Orthodontics, Technische Universität Dresden, Germany \\ ${ }^{4}$ Department of Oral Surgery, Wroclaw Medical University, Poland \\ A - research concept and design; B - collection and/or assembly of data; $\mathrm{C}$ - data analysis and interpretation; \\ $D$ - writing the article; $E$ - critical revision of the article; $F$ - final approval of the article
}

\section{Address for correspondence \\ Monika Machoy \\ E-mail:m.machoy@gmail.com}

\section{Funding sources}

None declared

\section{Conflict of interest}

None declared

Received on February 6, 2019

Reviewed on June 23, 2019

Accepted on December 5, 2019

Published online on March 24, 2020

\begin{abstract}
Innovative computer techniques are starting to be employed not only in academic research, but also in commercial production, finding use in many areas of dentistry. This is conducive to the digitalization of dentistry and its increasing treatment and diagnostic demands. In many areas of dentistry, such as orthodontics and maxillofacial surgery, but also periodontics or prosthetics, only a correct diagnosis ensures the correct treatment plan, which is the only way to restore the patient's health. The diagnosis and treatment plan is based on the specialist's knowledge, but is subject to a large, multi-factorial risk of error. Therefore, the introduction of multiparametric pattern recognition methods (statistics, machine learning and artificial intelligence (Al)) is a great hope for both the physicians and the patients. However, the general use of clinical decision support systems (CDSS) in a dental clinic is not yet realistic and requires work in many aspects - methodical, technological and business. The article presents a review of the latest attempts to apply Al, such as CDSS or genetic algorithms (GAs) in research and clinical dentistry, taking under consideration all of the main dental specialties. Work on the introduction of public CDSS has been continued for years. The article presents the latest achievements in this field, analyzing their real-life application and credibility.
\end{abstract}

Key words: dentistry, clinical decision support systems, machine learning, artificial intelligence, CDSS

Cite as

Machoy ME, Szyszka-Sommerfeld L, Vegh A, Gedrange T,

Woźniak K. The ways of using machine learning in dentistry.

Adv Clin Exp Med. 2020;29(3):375-384.

doi:10.17219/acem/115083

DOI

10.17219/acem/115083

\section{Copyright}

Copyright by Author(s)

This is an article distributed under the terms of the

Creative Commons Attribution 3.0 Unported (CC BY 3.0)

(https://creativecommons.org/licenses/by/3.0/) 


\section{Background}

Digitalization in dentistry has increased significantly over the last 10-20 years. In most developing countries, the shortage of medical and dental professionals stimulates the need for technology, especially artificial intelligence (AI) software. This can reduce costs, time, the need for human knowledge, and the number of medical errors.

Applications in the field of dental science vary according to needs - from dental emergencies, through differential diagnosis of pain in the mouth, interpretation of radiographic images, analysis of facial growth in orthodontics, to planning the optimal prosthetics for a particular patient. Despite the recognized demand for clinical decision support systems (CDSS), the production of these systems has been limited and slow to date. This can be due to the lack of formal evaluation of the systems, challenges in programming development, cost, and skepticism about the value and feasibility of CDSS. The use of digitalization technology in dental practice has grown rapidly in recent years. It is hoped that the incidence of dental and periodontal diseases as well as deaths related to oral and maxillofacial diseases will be reduced, making it easier to take care of the patient.

According to Mendonça, ${ }^{1}$ CDSS can be divided into several basic groups depending on the applied data analysis technique. The types of these systems are listed in Table 1.

This paper presents the general assumptions of the expert systems in dentistry and the latest 10 -year results of research published in all fields of dentistry, based on the medical databases of MEDLINE (PubMed) and Dentistry \& Oral Sciences Source (EBSCO).

\section{Applications of expert systems in different dental specializations}

The topics in which CDSS have been used are systematized in Table 2. Every topic has been extended in the text below.

\section{Orthodontics}

The decision to remove teeth for orthodontic treatment is important and difficult, because it tends to rely on the physician's experience. An AI expert system has been developed to diagnose extraction using the neuronal network (NN; type of machine learning), and to evaluate the effectiveness of this model. Using the back-propagation (BP) algorithm, 4 models of NN learning were constructed and evaluated for the diagnosis of extraction. Success and classification accuracy on the studied models were 93\% for extraction diagnostics compared to non-extraction diagnostics, and $84 \%$ for detailed diagnosis of extraction patterns. Despite many limitations of this study (only a small, exact group of cases could be examined), the results suggests that orthodontic systems may be useful in the case of expert systems with machine learning in NN which would be a great help for the less practiced clinicians.

Table 1. Types of clinical decision support systems (CDSSs) in medicine

\begin{tabular}{|c|c|c|}
\hline $\begin{array}{l}\text { Type of clinical decision } \\
\text { support systems }\end{array}$ & Mode of action & Examples of first applications \\
\hline Algorithmic systems & $\begin{array}{l}\text { Used in logical classification methods, } \\
\text { represented as DTs and flowcharts that } \\
\text { lead the user to a desired endpoint. This } \\
\text { approach does not depend on large } \\
\text { sample sizes of data and can be applied } \\
\text { across patient populations. }\end{array}$ & $\begin{array}{l}\text { - recommendation of chemotherapy drugs for breast cancer }{ }^{2} \\
\text { - a diagnostic aid for oral pathology } 3,4\end{array}$ \\
\hline Neural networks & $\begin{array}{l}\text { Algorithms that require training to create } \\
\text { a set of solutions to a problem. }\end{array}$ & $\begin{array}{l}\text { - first implemented in the } 1940 s^{5} \text { as a biological model of the brain } \\
\text { - particularly successful at narrow and well-defined clinical problems such } \\
\text { as classifying textual output of images diagnosis support }{ }^{6-9} \text { and prognosis } \\
\text { evaluation }{ }^{10,11} \\
\text { - commercialized for image recognition and used in uterus cervix cytology labs } \\
\text { - } \text { applied in dentistry to identify people at risk of oral cancer and pre-cancer }{ }^{12} \\
\text { - also tested in lower third molar treatment planning decisions }{ }^{13}\end{array}$ \\
\hline Probabilistic systems & $\begin{array}{l}\text { Incorporate rates of diseases or problems } \\
\text { in a population and the likelihood } \\
\text { of various clinical findings in order } \\
\text { to calculate the most likely explanation } \\
\text { for a particular clinical case. }\end{array}$ & $\begin{array}{l}\text { - Oral Radiographic Differential Diagnosis (ORAD), a program to assist in oral } \\
\text { radiographic diagnosis }{ }^{14} \text { and a system that assists pulpal diagnosis }{ }^{15}\end{array}$ \\
\hline $\begin{array}{l}\text { Logical/deductive } \\
\text { systems }\end{array}$ & $\begin{array}{l}\text { Branching logic - a collection of if-then } \\
\text { rules - to make decisions. }\end{array}$ & - RHINOS, a consultation system for diagnosis of headache and orofacial pain ${ }^{16}$ \\
\hline Critiquing model & $\begin{array}{l}\text { Program that reacts to proposed } \\
\text { diagnosis or treatment with agreement } \\
\text { or alternatives. }\end{array}$ & $\begin{array}{l}\text { - ATTENDING, HyperCritic and RaPiD. Both HT-ATTENDING }{ }^{17} \text { and HyperCritic }{ }^{18} \\
\text { are systems designed to critique the management of hypertensive patients. } \\
\text { RaPiD uses both an automated and critiquing model for removable partial } \\
\text { denture design }{ }^{19}\end{array}$ \\
\hline Hybrid systems & $\begin{array}{l}\text { Combine both deductive rules and } \\
\text { probabilistic reasoning in the same CDSS. }\end{array}$ & - HEME, a system used to diagnose blood diseases in 1950s \\
\hline
\end{tabular}


Table 2. Application of CDSS in various dental fields

\begin{tabular}{|c|c|}
\hline Dental specialization & Application of CDSS \\
\hline Orthodontics & $\begin{array}{l}\text { - the diagnosis of extractions with NN machine learning }{ }^{20} \\
\text { - computational formulation of orthodontic tooth-extraction decisions }{ }^{21} \\
\text { - design and implementation of a hybrid GA and ANN system for predicting the sizes of unerupted canines and premolars } 22 \\
\text { - ANN modeling for deciding if extractions are necessary prior to orthodontic treatment }{ }^{23} \\
\text { - factors affecting the clinical approach to impacted maxillary canines }{ }^{24} \\
\text { - paraconsistent ANN as auxiliary in cephalometric diagnosis }{ }^{25} \\
\text { - use of automated learning techniques for predicting mandibular morphology in skeletal class I, II and III26 } \\
\text { - an automatic method for skeletal patterns classification using craniomaxillary variables on a Colombian population }{ }^{27}\end{array}$ \\
\hline $\begin{array}{l}\text { Conservative dentistry } \\
\text { and prosthodontics }\end{array}$ & $\begin{array}{l}\text { - modeling the longevity of dental restorations by means of a CBR system }{ }^{28} \\
\text { - the prediction in computer color matching of dentistry based on GA+BP NN }{ }^{29} \\
\text { - an ontology-driven, case-based clinical decision support model for removable partial denture design }{ }^{30} \\
\text { - decision support system for predicting color change after tooth whitening }\end{array}$ \\
\hline Periodontology & $\begin{array}{l}\text { - diagnosis of periodontal diseases using different classification algorithms (a preliminary study) }{ }^{32} \\
\text { - ANNs for the diagnosis of aggressive periodontitis trained with immunologic parameters } \\
\text { - supervised machine learning-based classification of oral malodor based on the microbiota in saliva samples }{ }^{34} \\
\text { - predicting recurrent aphthous ulceration using GA-optimized NNs }{ }^{35}\end{array}$ \\
\hline $\begin{array}{l}\text { Temporomandibular } \\
\text { joint disorders }\end{array}$ & $\begin{array}{l}\text { - BBN analysis applied to determine the progression of TMD using MRI }{ }^{36} \\
\text { - outcome of } 3 \text { screening questions for TMD (3Q/TMD) on clinical decision-making }{ }^{37} \\
\text { - use of ANN in differentiation of subgroups of temporomandibular internal derangements (a preliminary study) }\end{array}$ \\
\hline Endodontics & $\begin{array}{l}\text { - the reliability of ANN in locating minor AF (a cadaver study) }{ }^{39} \\
\text { - a new approach for locating the minor AF using ANN }\end{array}$ \\
\hline Dental surgery & $\begin{array}{l}\text { - performance of ANN for vertical root fracture detection (an ex vivo study) }{ }^{41} \\
\text { - CDSS for dental treatment }{ }^{42} \\
\text { - CDSS in dental implantology }\end{array}$ \\
\hline Maxillofacial surgery & $\begin{array}{l}\text { - oral cancer prognosis based on clinicopathologic and genomic markers using a hybrid of feature selection and machine } \\
\text { learning methods }{ }^{44} \\
\text { - ANN analysis to assess hypernasality in patients treated for oral or oropharyngeal cancer }{ }^{45} \\
\text { - application of FL in oral cancer risk assessment }{ }^{46}\end{array}$ \\
\hline
\end{tabular}

Improved performance has been achieved with elements such as proper selection of input data, appropriate organization of modeling and preferred generalization. ${ }^{20}$

The topic of decision support for orthodontic extraction was also raised several years earlier. The authors developed a mathematical model that simulates whether or not to extract teeth in optimizing orthodontic treatment outcome and formulates the morphologic traits sensitive to optimizing the tooth-extraction/non-extraction decisions. Orthodontic records of patients with good treatment outcomes were collected, and dentofacial morphologic traits, along with their degrees of influence in the optimized model, were determined. The rate of coincidence between the recommendations given by the optimized model and the actual treatments performed was found to be $90.4 \% .^{21}$

Also, other studies have been published in the field of orthodontics. They aimed at developing a hybrid genetic algorithm (GA) and artificial NN (GA-ANN) to evaluate the size of canine teeth and premolars that did not emerge during tooth replacement. The data was derived from measurements of tooth models. The GA-ANN hybrid algorithm was used to find the best reference teeth and the most accurate mapping function. During each iteration, the GA introduced the reference teeth into the ANN. The ANN tried to find the best mapping function for relating the reference inputs to the targets. Next, the algorithm checked the stopping criteria. If satisfying, the results were reported; if not, the GA moves to the next generation, searching for better possible candidates among the reference teeth. This process was repeated until the algorithm found a result that satisfied the stopping criteria or until the number of generations exceeded the predefined value. ${ }^{22}$ The percentage of predictive errors and the ratios of over- or underestimation when using the GA-ANN hybrid algorithm were lower than in the case of linear regression analysis. The method is very promising, but was only tested in 1 ethnic group. More generalized studies in different ethnic groups are needed to validate the feasibility of the proposed method. There are still no updates of the method.

The experiment in orthodontics was aimed at building a decision-making expert system for the treatment of orthodontic patients aged 11-15 years in order to determine using ANN whether extraction is necessary. In particular, factors affecting the decision-making process were discovered. The ANN designed in this study was $80 \%$ effective. $^{23}$ This BP ANN employs the error BP learning algorithm. The basic principle of the $\mathrm{BP}$ algorithm is the propagation of errors from the output layer backward to the input layer by each layer that shares the error with neurons of each layer. The 20 test samples proved successful in evaluating factors that affect the decision-making process.

A very interesting and complicated topic about impacted canines from the border of orthodontics and dental surgery is discussed in the article by Nieri et al. ${ }^{24}$ The aim of the study was to apply Bayesian networks (statistics) 
to evaluate the relative role and possible causal relationships among various factors affecting the diagnosis and final treatment outcome of impacted maxillary canines. The demographic, orthodontic and periodontal variables were recorded and analyzed by means of NN. The network identified possible relationships among the variables considered for diagnosis and treatment of impacted canines. It confirmed the results of previous investigations on the same population in which the final periodontal outcomes after the surgical-orthodontics repositioning of maxillary impacted canines were unrelated to pretreatment diagnostic variables on the panoramic radiographs. ${ }^{25}$

Another work dealing with the subject of diagnosis in orthodontics presents the application of the paraconsistent artificial neural network (PANN) in the analysis of cephalometric variables and provides an orthodontic diagnosis. Patient input cephalometric values were compared with means drawn from individuals considered normal from the cephalometric point of view by the PANN. The analysis was targeted to measure skeletal and dental discrepancies and establish a cephalometric diagnosis. The analysis results were expressed in degrees of skeletal, anteroposterior, and dental discrepancy, pertinent to upper and lower incisors. A sample of 120 orthodontic patients was processed by the proposed model and 3 orthodontic experts. Comparisons between the model and the human experts' performance provided kappa indexes that varied from moderate to almost perfect agreement. The agreement between the model and specialist's performance was equivalent. In addition, the model pointed out contradictions presented in the data that were not noticed by the orthodontists, which highlights the contribution that this kind of system could make in orthodontics decision support. ${ }^{26}$

Niño-Sandoval et al. ${ }^{27}$ used an already existing machine learning software (as the only one of the articles described). RapidMiner predicts the mandibular morphology through craniomaxillary variables on lateral radiographs in patients with skeletal class I, II and III. The researchers use 2 machine learning techniques - ANN and support vector regression (SVR). Standardized lateral radiographs were used to create mandibular measurements. They were evaluated through a correlation coefficient using a ridge regression between the real value and the predicted value. The authors came to a conclusion that the used craniomaxillary variables showed a high predictability ability of the selected mandibular variables, which may be the key to facial reconstruction from specific craniomaxillary measures in the 3 skeletal classifications. The ANN compared with the SVR had a better performance and classification accuracy reflected in its higher coefficients. ${ }^{27}$

Despite the fact that the abovementioned algorithms were very promising in the orthodontic diagnosis, none of them were introduced into general orthodontic practice.

\section{Conservative dentistry and prosthodontics}

The lifespan of dental restorations is limited. Longevity depends on the material used and different dental characteristics. One recent study by Aliaga et al., ${ }^{28}$ based on a set of data from graphs, notes and radiological information from information analysis performed using AI, attempted to determine the most suitable material for the restoration of cavities and long-term monitoring of the reconstruction process. In order to classify the cases, a multilayer perceptron and a mixture of experts evaluated the data. The multilayer perceptron uses a NN which facilitates the combination of outputs obtained by both methods and reduces error. As shown, the output of the classifiers corresponds to the input of NN. The 2 classifiers were combined by means of NN. The conclusion of the presented study suggests that the system enables us to determine the type of reconstruction best suited to the patient, predicting the longevity of each procedure. The system adapts to new cases that are acquired by incorporating new data into the system database and updating information which is then used for new prognoses. This is an important issue which can lead to improved results in new cases introduced into the system. The technology has not been transferred to the clinical usage, though. ${ }^{28}$

The material needed to fill or replace the defect is just as important to the patient as the color of the selected filling or prosthetic restoration. Although the use of computer-based color matching can reduce the impact of subjective factors, matching the color of the natural tooth to ceramic one is still one of the most demanding topics in esthetic dentistry. Back propagation of neural networks (BPNN) has already been introduced to computer color matching in dentistry, but it has disadvantages such as instability and low accuracy. The study used GA to optimize the initial mass and threshold values in BPNN in order to improve the precision of matching. In accordance with expert knowledge, BPNN was combined with GA as a novel method for computer color matching in dentistry. Experiments have shown that the proposed method improves the precision and predictability of color matching in complementary dentistry. The existing improved BPNN has a low convergence rate and it is difficult to devise a suitable network structure. However, it has high practical application value and, with the development of computer science, dental practices will have more ability to provide better services for patients in the future. ${ }^{29}$

Also, in the field of prosthodontics, an initial study by Chen et al. was published ${ }^{30}$ that presented a CDSS model for specific design of removable partial dentures (RPDs). The authors developed an ontological paradigm to represent knowledge of patient's oral conditions and denture component parts as well as a cosine similarity algorithm to calculate similarity values between input patients and standard ontology cases. All the similarity metrics described in the article demonstrated the efficiency 
of the model. In conclusion, the authors admitted that the methodology merits further research development to match clinical applications.

The use of CDSS was also introduced in predicting the color of the teeth after the bleaching procedure. The authors implemented the CDSS using the regression model as an intelligent part of the system. The system consisted of 3 parts: patient individual data, pre-bleaching color input, and prediction of post-treatment color (output). The results obtained have demonstrated that the CDSS can predict the color change obtained with an in-office whitening system using colorimetric values. ${ }^{31}$

\section{Periodontology}

An attempt to apply AI was also made in the field of periodontal diseases - in periodontology. In recent studies, an identification unit has been developed for the classification of periodontal diseases using SVM, decision trees (DT) and NN. Patients were divided into 2 groups. The codes created for risk factors, data related to the periodontium and, radiographically, to bone loss were created as a matrix and treated as input data for the classification unit. In total, 6 conditions of the periodontium were the results of the classification unit. The accuracy of the proposed methods was compared according to their resolution and operation time. The DTs and SVM achieved the best accuracy rate at classifying periodontal diseases with high accuracy depending on clinical trials. The SVM and DT results were $98 \%$ with a total calculation time of $19.91 \mathrm{~s}$ and $7.00 \mathrm{~s}$, respectively. The worst correlation between input and output variables was for ANN, and its efficiency was estimated at $46 \%$. The SVM and DT proved to be complex enough to reflect all factors associated with the periodontal condition, and simple enough to be understandable and useful as a decision-making aid for predicting periodontal diseases. The program offered a supportive diagnostic tool for periodontal diseases with high accuracy and opened a new area for identifying periodontal diseases. However, further research with a wider population is recommended, including research on advanced algorithmic models that use clinical and imaging data. ${ }^{32}$

Tests were also carried out in the field of periodontology using ANNs to diagnose and treat aggressive periodontitis (AgP) resulting from autoimmune diseases. To date, no clinical, microbiological or histopathological markers or their combination have been developed to identify AgP in patients with chronic periodontitis (CP). The aim was to determine the probability density function of clinical and immunological data sets from patients with periodontal diseases and develop NNs to correctly classify patients into either AgP or CP class. Matching probability distributions to data sets was examined by means of the Akaike information criterion (AIC). The ANNs were trained by cross-entropy (CE) values estimated on the basis of the probability of demonstrating certain levels of immune parameters and the probability of the reference mode proposed by the kernel density estimation (KDE). In the case of ANNs, the weight distribution regularization parameter of method was determined using tenfold cross-validation. Possible evidence for 2 clusters of patients in cross sections and longitudinal weight loss measurements was revealed using KDE. Data sets with CD4/CD8 ratio showed from 2 to 7 clusters, CD3, monocytes, eosinophils, neutrophils, and lymphocytes, levels of interleukin (IL)-1, IL-2, IL-4, interferon gamma (INF- $\gamma$ ) and tumor necrosis factor $\alpha$ (TNF- $\alpha$ ) from monocytes, as well as antibody levels against Actinobacillus actinomycetemcomitans (Aa) and Porphyromonas gingivalis (Pg). The ANNs gave 90-98\% accuracy in classifying patients into both groups. The best overall predictor was ANN from CE monocytes, eosinophils, neutrophils, and CD4/CD8 as inputs. The ANNs may be invaluable in classifying periodontal patients into AgP or CP when they are supplied with CE values based on KDE. Therefore, ANNs can be used to accurately diagnose AgP or CP with relatively simple and conveniently obtained parameters, such as the peripheral blood leukocyte count. This will enable clinicians to better tailor specific therapeutic protocols for patients with AgP and CP. The algorithm can effectively classify periodontitis patients. Future work should be anticipated on bigger samples, extending the results of the present study and employing a wider array of parameters that can turn personalized treatment of periodontitis from concept to reality. ${ }^{33}$

In the field of periodontology and mucosal diseases, studies have been conducted on the composition of saliva and microorganisms in the mouth. Recent studies have shown an effective method for classifying yeasts from salivary microorganisms using SVM, ANNs and DTs. This approach uses the concentration of methyl mercaptan in the oral air as an indicator of oral odor and the peak areas of restriction fragment length polymorphisms (T-RF) of the $16 S$ rRNA gene as data for supervised machine learning methods without identifying specific species producing inflammatory oral compounds. Using T-RF proportions and frequencies, models have been developed to classify the presence of methyl mercaptan, volatile sulphur-containing compounds that cause yeasts in the oral cavity. The SVM classifiers successfully classify the presence of highly specific methyl mercaptan, and it is expected that such classification will be useful for saliva screening in respiratory tract allergies prior to visits to specialist clinics. Classification using SVM and ANN does not require the identification of oral species of microorganisms responsible for halitosis, and ANNs do not require T-RF proportions. ${ }^{34}$ Interesting findings from ANN studies, also in the field of mucosal diseases, presented in publication by Dar-Odeh et al., ${ }^{35}$ provide an opportunity to develop and optimize $\mathrm{NN}$ that can predict the occurrence of recurrent rheumatoid arthritis ulcers (RAU) based on a set of relevant input data. Artificial 
neural networks that take advantage of GA to optimize NNs architecture were used. Input and output data from 86 subjects (predisposing factors and participants' status for recurrent aphthous ulceration) were used to construct and train NNs. Optimized NNs were tested using unqualified data from 10 subsequent subjects. Based on data analysis, ANN found significant correlations between specific environmental, individual and behavioral factors and the occurrence of recurrent aphthosis. This is of great importance for screening patients and for the proper education of the exposed group.

\section{Temporomandibular joint disorders}

The latest AI technology has also been used to help in extremely complicated joint diseases in terms of analysis, diagnosis and treatment. This study investigated the analysis of magnetic resonance imaging (MRI) results to diagnose temporomandibular joint disorders (TMD). The purpose of the study was to determine the progression of TMD, focusing on how each discovery affects the others.

Briefly, 1.5-T MRI (33 variables) and diagnosis (bone changes and disk displacement) of patients with TMD in the years 2007-2008 were selected. The data was modified according to the TMD diagnostic criteria. Bayesian belief network (BBN) accuracy was compared using 11 algorithms (necessary path condition, path condition, greedy search-and-score with Bayesian information criterion, Chow-Liu tree, Rebane-Pearl polytree, tree-augmented naïve Bayes model, maximum log likelihood, Akaike information criterion, minimum description length, K2, and C4.5), multiple regression analysis, and an ANN with the use of usefulness validation and tenfold crossvalidation. The BBN path condition algorithm was $>99 \%$ accurate when using the regression validation and tenfold cross-validation. The BBN may represent cause-effect relationships between different results and attribute conditional probabilities that can then be used to interpret TMD progression. The research was made on the great dataset and the results are accurate. The program results would be helpful in clinical practice, especially when dealing with such a difficult topic. Effort should be made to implement the program in dental practice. ${ }^{36}$

The solution published by Lövgren et al. ${ }^{37}$ also helps in the field of TMD. The aim of the study was to determine the use of ANN to predict 2 subgroups of internal changes in temporomandibular joints (TMJ) and normal joints using characteristic clinical signs and symptoms. Clinical symptoms and diagnosis of 161 patients with temporomandibular joint internal derangement (TMJID) were considered a golden standard and were used to train NNs. After training, symptoms and the diagnosis of 58 new patients were used to test the ability of the network to diagnose. The diagnosis from ANN was compared with the diagnosis of a surgeon experienced in TMD. The sensitivity and specificity of ANN in predicting TMJ ID subtypes were assessed using clinical diagnosis as a golden standard. Eight cases assessed as bilaterally correct in a clinical trial were found to be normal by ANN. In the detection of unilateral anterior disc displacement with reduction (ADDwR), the sensitivity and specificity of ANN were 80\% and $95 \%$, respectively. In detecting unilateral anterior disc displacement without reduction, sensitivity and specificity of ANN were $69 \%$ and $91 \%$, respectively. In bilateral ADDwR detection, sensitivity and specificity of ANN were $37 \%$ and $100 \%$, respectively. In the case of bilateral ADDwRn, sensitivity and specificity of ANN were 100\% and $89 \%$, respectively. In detection of ADDwR on one side and ADDwR on the other side, sensitivity and specificity of ANN were $44 \%$ and $93 \%$, respectively.

Great progress in the use of CDSS has been made by Bas et al., ${ }^{38}$ who used it practically in a clinical screening study of patients with TMD. Considering factors such as gender, age, dental pain, 300 TMD-positives and 500 TMD-negatives were randomly selected from the study population (SPSS v. 22 for Macintosh; IBM Corp., Armonk, USA; random numbers) who needed or did not need TMD treatment. On the basis of the CDSS analysis, conclusions have been drawn which factor the influence on the higher risk of developing TMD (there was no difference in the occurrence of dental care or in the rate of subscription of dental care). Among TMD-positives, there was no significant difference in the proportion of women and men receiving treatment related to TMD. No significant association was found between which fee systems the individual belonged to and any specific type of treatment, and no significant association with TMD treatment for the independent variables of sex, age, profession of the examiner, or dental care subscription. The research was one of the first real clinical and practical uses of the CDSS in dentistry.

\section{Endodontics}

Artificial intelligence has also been introduced into the field of endodontics. The accuracy of ANN was evaluated in an attempt to simulate the clinical evaluation of the working length of the root canals of human teeth. It was measured on 50 extracted teeth by an endodontist. The working length was confirmed using radiography. The position of the endodontic file relative to the minor apical foramen (AF) was classified as distant, close and accurate by ANN, by endodontist prior to extraction and using stereomicroscope after extraction. There were significant differences between the data obtained by endodontists and ANN and the data obtained by endodontists and actual measurements with a stereomicroscope after extraction. The evaluation of endodontists was accurate in $76 \%$ of the teeth. The ANN found correct anatomic position in $96 \%$ of the teeth and was more accurate than endodontic markings compared to actual working length measurements using a stereomicroscope as a golden standard after tooth extraction. An ANN can be used 
to exactly determine the working length, which will be of great importance in clinical practice and treatment success as well as in the long-term maintenance of root canal-treated teeth. ${ }^{39}$

A very similar study, conducted in the same year and in the same research center, presented a new approach to finding minor AF. ${ }^{40}$ The new approach was developed to locate AF using feature extraction from radiography, followed by data processing using ANN as CDSS. Fifty straight single-rooted teeth placed in the alveolar process of the jaw of a dissected skull were selected. Access to the chamber and canal of the tooth was established and the file was placed in the canals in order to determine the working length. A radiograph was performed to evaluate the location of the file relative to AF and the location was re-evaluated after tooth removal. The file end location was split identically to the previously described experiment. Each radiograph was used to extract relevant functions using unsupervised machine learning method K-means for clustering, Otsu's method and the Wavelet protocol. Thirty-six functions were used for training, and the others were used to evaluate the multilayer perceptron ANN model. Analysis of radiographic images (test samples) using ANN showed that in $93 \%$ of the samples, AF location was correctly determined by false rejection and acceptance of errors. It has been shown that ANNs may be the second opinion in locating AF on radiographs to increase the accuracy of radiography in determining the working length. In addition, ANN can function as CDSS in various similar clinical situations.

\section{Dental surgery}

A study presenting NN developed for the detection of vertical root fractures may be also placed in the field of endodontics or at the border of dental surgery. This is an important and difficult issue because vertical fractures are often difficult to diagnose by a clinician due to cracks that overlap anatomical structures in $2 \mathrm{D}$ dental X-rays.

A probabilistic NN design was used to assess whether the root of the tooth was healthy or had a vertical fracture. Two hundred photos from digital radiography - used to train and test NNs - were divided into 3 groups according to the number of training and test sets. Both the training data and the test data were evaluated using gray data on the line passing through the root. The function deviation in the reconnaissance data was estimated between 0 and 1 to select the best NN performance. Neural network results were evaluated using a diagnostic test. The NN designed in this study had sufficient sensitivity, specificity and accuracy to be a model for detecting vertical root fractures. However, before extending to a clinical application, further research is needed in order to work around several clinical problems, i.e., the grey-scale data in the experimental root fracture without any radio density material certainly differ from the variety of root canals in clinical cases. ${ }^{41}$

Another article connected to dental surgery has been published by Mago et al. ${ }^{42}$ The authors proposed an expert system based on fuzzy logic (FL) that was supposed to help to decide which treatment should be introduced in the case of the broken tooth.

Fuzzy logic is a form of AI that uses a collection of membership functions and fuzzy rules, instead of traditional bivalued logic. Fuzzy logic systems are capable of dealing with imprecise values or vague concepts and are able to imitate human reasoning capabilities, which must deal with imprecise or not-well-defined terms. The authors concluded that the system is identical to dentists' predictions with respect to treatments. The system has been rigorously tested and the predictions are consistent with those supplied by the tested dentists, so it is concluded that the system is operating similarly to the intelligent behavior of the dentists. This system can be treated as an expert "second opinion" to help dentists during the decision-making process of choosing treatment for a cracked or broken tooth. However, the software still remains only in the academic usage.

The field of dental surgery includes implantology, which merges prosthetics, orthodontics and periodontology. Therefore, the clinician needs to analyze huge amount of data before the decision about implantation. The article by Polášková et al. ${ }^{43}$ presents a web application that provides recommendations for application of the implant based on anamnesis and medical examination such as 3D measurements, diagnostic information for treatment planning and objective measurement of implant placement. The core of the program concerned expert knowledge programming, such as DT. The structure model had 4 basic components: inference engine, knowledge base, working memory, and explanation. This CDSS is based on the comprehensive appraisal of the morphologic features of the proposed implantation site and can practically help to formulate an objective treatment plan in general practice, one that would include implant objectification. The system would be greatly helpful for dentists as long as it would be widely available and widespread.

\section{Maxillofacial surgery}

The field of dentistry that is particularly important in everyday practice is maxillofacial surgery, which involves, i.a., the diagnosis and treatment of oral cancer. A potential diagnostic error may cost the life of patients. Therefore, it would be advisable to introduce objective solutions, allowing for $100 \%$ correct diagnosis. Chang et al. ${ }^{44}$ focused on this problem. The main aim of this research was to apply a hybrid of feature selection and machine learning methods in oral cancer prognosis based on the parameters of the correlation of clinicopathologic and genomic markers. In the $1^{\text {st }}$ stage of this study, 5 methods of feature 
selection were proposed and a set of data on oral cancer prognosis was experimented with. In the $2^{\text {nd }}$ stage, the model with the functions selected from each feature selection method was tested on the proposed classifiers. Four types of classifiers were selected, namely adaptive neuro-fuzzy inference system (ANFIS), NN, SVR, and logistic regression. Results have shown that prognosis is superior to the presence of clinical and genomic markers. The research was conducted on a small sample size mostly because of the medical confidentiality problems. Some patients, especially with such a difficult health problem as cancer, do not wish to reveal any information about their diseases to others, and are unwilling to donate their tissues for research/educational purposes. As for clinicians, some may not want to share patients' data with others, especially those from the non-medical fields, while some do not keep their medical records in the correct medical form. Among the available cases, some patients' clinicopathologic data were incomplete, some tissues were missing due to improper management and some were duplicated cases. Due to that, the number of cases that could actually be used for this research was very limited. In order to overcome the problem, the authors employed the feature selection methods on their dataset to choose the most optimal feature subsets based on the correlations of the input and output variables. The features selected were fed into the proposed classifier. The method is very promising and should be extended to as many clinical centers as possible. ${ }^{44}$

Neural network analysis has been also used to assess hypernasality in patients treated for oral or oropharyngeal cancer. The researchers investigated the applicability of NN feature analysis of nasalance in speech to assess hypernasality in the speech of patients treated for the abovementioned cancers. The speech recordings were evaluated regarding hypernasality, articulation, intelligibility, and patient-reported speech outcome. Objective measurement with ANN was not able to differentiate between patient for tumor stage and tumor location, while trained listeners are able to differentiate between patients for tumor stage, but not for tumor location. In conclusion, ANN is not yet able to substitute trained raters. ${ }^{45}$

The topic of the risk and causes of the malignization is presented in a recently published article by Scrobotă et al., ${ }^{46}$ in which the authors aimed to estimate the oxidative stress related-cancerization risk of the potentially malignant processes. They used FL to interpret the values in the input data and to assign values to the output in order to implement the multi-criteria CDSS. The risk was estimated as a concrete numerical value on a scale from 1 to 10 depending on the input numerical/linguistic value. The system can be a great achievement in oral cancer screening. However, it needs to be tested on a greater sample (in the research, only 16 samples were tested) before it could be admitted as a reliable instrument to infer the screening in this very challenging field of medicine.

\section{Conclusions and comments}

1. As in another disciplines, SVM brings the best accuracy and performance because of method construction. However, the articles present the contemporary achievements in CDSS without their critical assessment, largely displaying a very optimistic approach to the subject.

2 . The validity of CDSSs is mostly established in narrow domains under varying conditions and technologies. Most of the systems have not been formally evaluated, and their value for clinical practice could not be established. Currently, CDSSs, in general, are developing as fragments and isolated systems with a few clinic- or hospital-wide exceptions in academic centers.

3. All of the algorithms and projects presented in the review have been validated only internally. The algorithms use small, inner databases. None of them have been shown to affect real clinical decision-making.

4. A small sample size is a common problem associated with medical datasets. The costs that have to be incurred and the usually inconsistent, incomplete or noisy samples make it almost impossible to gather adequate samples. The small sample size problem is mostly visible in the oral cancer research.

5. The validation of the datasets is not performed truly independently. The cross-validation method is used, which might have too many variables that can improperly modify the developing and training of the classifier, which is why it is necessary to assess its performance on completely independent datasets, preferably from different institutions.

6. The presented ideas have a great potential to be a very helpful key for the clinicians to shorten the diagnostics and make it simpler and more reliable.

7. To successfully implement CDSSs in dental practice, there is a need to overcome many practical difficulties:

a) A dentist's work is rarely concentrated around a university clinic, where there are a few dentists for 1 program; dentists have their own private practice, often one-person. This means that the CDSSs would have to be financially available for the average dentist.

b) Only a few dentists specialize in one area, such as periodontics, surgery, orthodontics, or endodontics. Most of them perform treatments in many fields of dentistry, which means that a very extensive program should be created, making decisions in a very wide range of areas.

c) Ideally, the program should be immediately linked to an electronic patient database, so that the physician only writes data from the interview and clinical trial - otherwise the program would take too much time in the chair and it would not be cost-effective for the dentist. The "currency of the chair-time" refers to the time that a physician must devote to performing a particular procedure.

8. The focus should be on creating a precise and practical program in the most specialized and the most difficult areas of dentistry. This should occur primarily in academic hospitals, such as facial and maxillofacial surgery 
departments, especially for the assessment of the advancement and treatment of orthostatic neoplasms and orthognathic surgery, which are fields that link orthodontics and facial-jaw surgery, treating dentomandibular, mostly severe genetic malocclusions.

9. The impact of the abovementioned techniques in clinical dentistry is inconsiderable so far, but they are very promising in the future.

\section{ORCID iDs}

Monika Elżbieta Machoy (1) https://orcid.org/0000-0001-5787-222X Liliana Szyszka-Sommerfeld (1) https://orcid.org/0000-0002-1103-1297 Andras Vegh (1) https://orcid.org/0000-0002-2997-3805

Tomasz Gedrange (1) https://orcid.org/0000-0002-3551-6467

Krzysztof Woźniak (i) https://orcid.org/0000-0002-5088-8760

\section{References}

1. Mendonça EA. Clinical decision support systems: Perspectives in dentistry. J Dent Educ. 2004;68(6):589-597.

2. Wirtschafter D, Carpenter JT, Mesel E. A consultant-extender system for breast cancer adjuvant chemotherapy. Ann Intern Med. 1979;90(3): 396-401.

3. Rudin JL.DART (Diagnostic Aid and Resource Tool): A computerized clinical decision support system for oral pathology. Part 2. Compendium. 1995;16(1):8,10,12 passim; quiz 17.

4. Rudin JL. DART (Diagnostic Aid and Resource Tool): A computerized clinical decision support system for oral pathology. Compendium. 1994;15(10):1316,1318,1320 passim.

5. McKnight LK, Stetson PD, Chen ES, Cimino JJ. Improving clinical communication with a virtual whiteboard. Proceedings/AMIA Annual Fall Symposium, 2002

6. Cross SS, Harrison RF, Kennedy RL. Introduction to neural networks. Lancet. 1995;346(8982):1075-1079.

7. Rennels GD, Shortliffe EH. Advanced computing for medicine. Sci Am. 1987;257:154-161.

8. Shortliffe EH.Computer programs to support clinical decision making. JAMA. 1987;258(1):61-66.

9. Walton JD, Musen MA, Combs DM, Lane CD, Shortliffe EH, Fagan LM Graphical access to medical expert systems. III: Design of a knowledge acquisition environment. Methods Inf Med. 1987;26(3):78-88.

10. Rennels GD, Shortliffe EH, Stockdale FE, Miller PL. A computational model of reasoning from the clinical literature. Comput Methods Programs Biomed. 1987;24(2):139-149.

11. Heckerman DE, Horvitz EJ, Nathwani BN. Toward normative expert systems. Part l: The Pathfinder Project. Methods Inf Med. 1992;31(2):90-105.

12. Speight PM, Elliott AE, Jullien JA, Downer MC, Zakrzewska JM. The use of artificial intelligence to identify people at risk of oral cancer and precancer. Br Dent J. 1995;179(10):382-387.

13. Brickley MR, Shepherd JP. Performance of a neural network trained to make third-molar treatment-planning decisions. Med Decis Making. 1996;16(2):153-160.

14. White SC. Computer-aided differential diagnosis of oral radiographic lesions. Dentomaxillofac Radiol. 1989;18(2):53-59.

15. Hyman JJ, Doblecki W. Computerized endodontic diagnosis. J Am Dent Assoc. 1983;107:755-758.

16. Matsumura Y. RHINOS: A consultation system for diagnosis of headache and facial pain. Comput Methods Programs Biomed. 1986;23(1): 65-71.

17. Miller PL, Black HR. HT-ATTENDING: Critiquing the pharmacologic management of essential hypertension. JMed Syst. 1984;8(3):181-187.

18. van der Lei J, Musen MA. A model for critiquing based on automated medical records. Comput Biomed Res. 1991;24(4):344-378.

19. Davenport JC, Hammond P, Hazlehurst P. Knowledge-based systems, removable partial denture design and the development of RaPiD. Dent Update. 1997;24(6):227-233.

20. Seok Ki J, Tae-Woo K. New approach for the diagnosis of extractions with neural network machine learning. Am J Orthod Dentofacial Orthop. 2016;149(1):127-133. doi:10.1016/j.ajodo.2015.07.030
21. Takada K, Yagi M, Horiguchi E. Computational formulation of orthodontic tooth-extraction decisions. Part l: To extract or not to extract. Angle Orthod. 2009;79(5):885-891.

22. Moghimi S, Talebi M, Parisay I. Design and implementation of a hybrid genetic algorithm and artificial neural network system for predicting the sizes of unerupted canines and premolars. Eur J Orthod. 2012; 34(4):480-486. doi:10.1093/ejo/cjr042

23. Xie X, Wang L, Wang A. Artificial neural network modeling for deciding if extractions are necessary prior to orthodontic treatment. Angle Orthod. 2010;80(2):262-266. doi:10.2319/111608-588.1

24. Nieri M, Crescini A, Rotundo R, Bacetti T, Cortellini P, Pini Prato GP. Factors affecting the clinical approach to impacted maxillary canines: A Bayesian network analysis. Am J Orthod Dentofacial Orthop. 2010; 137(6):755-762. doi:10.1016/j.ajodo.2008.08.028

25. Mario MC, Abe JM, Ortega NR, Del Santo M Jr. Paraconsistent artificial neural network as auxiliary in cephalometric diagnosis. Artif Organs. 2010;34(7):E215-221. doi:10.1111/j.1525-1594.2010.00994.x

26. Niño-Sandoval TC, Guevara Pérez SV, González FA, Jaque RA, Infante-Contreras C. Use of automated learning techniques for predicting mandibular morphology in skeletal class I, II and III. Forensic Sci Int. 2017;281:187.e1-187.e7. https://doi.org/10.1016/j.forsciint.2017. 10.004

27. Niño-Sandoval TC, Guevara Perez SV, González FA, Jaque RA, InfanteContreras C. An automatic method for skeletal patterns classification using craniomaxillary variables on a Colombian population. Forensic Sci Int. 2016;261:159.e1-6. doi:10.1016/j.forsciint.2015.12.025

28. Aliaga IJ, Vera V, De Paz JF, García AE, Mohamad MS. Modelling the longevity of dental restorations by means of a CBR system. Biomed Res Int. 2015;2015:540306. doi:10.1155/2015/540306

29. Li H, Lai L, Chen L, Lu C, Cai Q. The prediction in computer color matching of dentistry based on GA+BP neural network. Comput Math Methods Med. 2015;2015:816719. doi:10.1155/2015/816719

30. Chen $Q$, Wu J, Li S, Lyu P, Wang Y, Li M. An ontology-driven, casebased clinical decision support model for removable partial denture design. Sci Rep. 2016;6:27855. doi:10.1038/srep27855

31. Thanathornwong B, Suebnukarn S, Ouivirach K. Decision support system for predicting color change after tooth whitening. Comput Methods Programs Biomed. 2016;125:88-93. doi:10.1016/j.cmpb.2015. 11.004

32. Ozden FO, Özgönenel O, Özden B, Aydogdu A, Niger J. Diagnosis of periodontal diseases using different classification algorithms: A preliminary study. Clin Pract. 2015;18(3):416-421. doi:10.4103/11193077.151785

33. Papantonopoulos G, Takahashi K, Bountis T, Loos BG. Artificial neural networks for the diagnosis of aggressive periodontitis trained by immunologic parameters. PLoS One. 2014;9(3):e89757. doi:10.1371/ journal.pone.0089757

34. Nakano Y, Takeshita T, Kamio N, et al. Supervised machine learningbased classification of oral malodor based on the microbiota in saliva samples. Artif Intell Med. 2014;60(2):97-101. doi:10.1016/j.artmed. 2013.12.001

35. Dar-Odeh NS, Alsmadi OM, Bakri F, et al. Predicting recurrent aphthous ulceration using genetic algorithms-optimized neural networks. Adv Appl Bioinform Chem. 2010;3:7.

36. Iwasaki H. Bayesian belief network analysis applied to determine the progression of temporomandibular disorders using MRI. Dentomaxillofac Radiol. 2015;44(4):20140279. doi:10.1259/dmfr.20140279

37. Lövgren A, Marklund S, Visscher CM, Lobbezoo F, Häggman-Henrikson $B$, Wänman A. Outcome of three screening questions for temporomandibular disorders (3Q/TMD) on clinical decision-making. J Oral Rehabil. 2017;44(8):573-579. doi:10.1111/joor.12518

38. Bas B, Ozgonenel O, Ozden B, Bekcioglu B, Bulut E, Kurt M. Use of artificial neural network in differentiation of subgroups of temporomandibular internal derangements: A preliminary study. J Oral Maxillofac Surg. 2012;70(1):51-59. doi:10.1016/j.joms.2011.03.069

39. Saghiri MA, Garcia-Godoy F, Gutmann JL, Lotfi M, Asgar K. The reliability of artificial neural network in locating minor apical foramen: A cadaver study. J Endod. 2012;38(8):1130-1134. doi:10.1016/j.joen. 2012.05.004

40. Saghiri MA, Asgar K, Boukani KK, et al. A new approach for locating the minor apical foramen using an artificial neural network. Int Endod J. 2012;45(3):257-265. doi:10.1111/j.1365-2591.2011.01970.x 
41. Kositbowornchai S, Plermkamon S, Tangkosol T. Performance of an artificial neural network for vertical root fracture detection: An ex vivo study. Dent Traumatol. 2013;29(2):151-155. doi:10.1111/j.16009657.2012.01148.x

42. Mago VK, Bhatia N, Bhatia A, Mago A. Clinical decision support system for dental treatment. J Comput Sci. 2012;3(5):254-261. https:// doi.org/10.1016/j.jocs.2012.01.008

43. Polášková A, Feberová J, Dostálová T, Kř́ž P, Seydlová M. Clinical decision support system in dental implantology. MEFANET J. 2013;1:11-14.
44. Chang SW, Abdul-Kareem S, Merican AF, Zain RB. Oral cancer prognosis based on clinicopathologic and genomic markers using a hybrid of feature selection and machine learning methods. BMC Bioinformatics. 2013;14:170. doi:10.1186/1471-2105-14-170

45. de Bruijn M, ten Bosch L, Kuik DJ, Langendijk JA, Leemans CR, Verdonck-de Leeuw I. Artificial neural network analysis to assess hypernasality in patients treated for oral or oropharyngeal cancer. Logoped Phoniatr Vocol. 2011;36(4):168-174.

46. Scrobotă I, Băciuț G, Filip AG, Todor B, Blaga F, Băciuț MF. Application of fuzzy logic in oral cancer risk assessment. Iran J Public Health. 2017;46(5):612-619. 